\title{
Analysis Tunneling IPv4 and IPv6 on VolP Network
}

\author{
Muhammad Ismu Haji ${ }^{* 1}$, Sugeng Purwantoro E. S. G. S $^{2}$, Satria Perdana Arifin ${ }^{3}$ \\ 1,2,3 Politeknik Caltex Riau \\ ismu14ti@mahasiswa.pcr.ac.id ${ }^{* 1}$, sugeng@pcr.ac.id², satria@pcr.ac.id ${ }^{3}$
}

\begin{abstract}
Using of IP addresses is currently still using IPV4. Meanwhile, the availability of the IPV4 address is gradually diminishes. IPv4 has a limited address capacity. IPv6 was developed with a capacity greater than IPv4. Connect between IPv4 and IPv6 without having to interfere with the existing infrastructure. So, methods like tunneling are needed. Tunneling builds a way that IPv4 and IPV6 can communicate. 6to4 tuning makes IPv6 able to communicate with IPV4 over IPV4 infrastructure. Real time communication is needed by internet users to be able to connect to each other. One of the real time communications is VolP. To find out the quality of tunneling implemented on a VolP network, it will analyze QoS such as delay, packet loss, and jitter. Delay obtained is 20,01 ms for IPv4, 19,99ms for IPv6 and 20,03ms for 6to4. Packet loss obtained 0,01\% for IPv4, IPv6 0,01\% and 6to4 0,08\%. The obtained jitter is 7,96ms for IPv4, IPv6 7.39ms, and 8,48 for 6to4. The test results show that using IPv6 gets a better QoS value than using IPv4 and 6 to 4 tunneling. The results using 6 to 4 tunneling obtained the highest QoS value between IPv4 and IPv6. Implementation using 6to4 tunneling results in high results because, IPv6 packets that are sent are wrapped into the IPv4 form to get through the IPv4 infrastructure.
\end{abstract}

Keywords: Tunneling, IPv4, IPv6, VoIP, Quality of Service

\section{Introduction}

The development of technology in computer networks are currenty growing rapidly. The development of technology in computer networks makes more users and and also easier to access. At present, everyone can connect to the internet and access anything. One of the technology continues to grow makes one able to communicate and exchange information using any device based on Internet Protocol (IP).

At this time development of technology has led to multimedia-based services or applications over real time IP networks. Because of this development, many IP-based multimedia services have emerged. One of services that allows communicating on an IP-based computer network is VolP.

There are two types of Internet Protocol (IP) addresses used by users, namely IP version 4 (IPv4) and IP version 6 (IPv6). IPv4 was developed in 1983 and it is still using today. IPv4 is 32bit based $(232$ or about $4,294,967,296)$ written in 4 octets and in dotted decimal notation. Since it was first introduced has helped every internet user around the world for more than last 20 years and it will not to be able to accommodate of using many internets addressing. The Internet Assigned Numbers Authority (IANA), an official international agency which manage IP addresses released data that the number of addresses remaining from IPv4 in 2011 was only about 10 percent of the total available or around 400 million addresses [1]. IPv6 is a protocol that has been designed by the IETF (Internet Engineering Task Force) to replace IPv4. IPv6 has an address capacity of 340 undecillion public addresses [2].

Basically, IPv6 doesn't compatible with IPv4, so a certain mechanism is needed IPv6 can connect with IPv4. The problem is how to make an infrastructure, so the two different IP versions can be connected and transfer data to each other. The need to exchange information is needed. But, the existing network infrastructure doesn't need to be change. The exist infrastructure that has used IPv4 and infrastructure using IPv6 can connect and exchange information each other.

There is a method to connecting these two different versions IP [3]. Basically, tunnelling just provide a tunnel for IPv6 packets via IPv4. One of tunnelling system is IPv6 tunnel broker which the tunnel is automatically activated by the tunnel broker to the isolated dual stack IPv6 / IPv4 host with another IPv6 network so that it can connect to the IPv6 network, through the existing IPv4 network.

Haji, M., E.S.G.S, S., \& Arifin, S. (2018). Analysis Tunneling IPv4 and IPv6 on VoIP Network. Kinetik: Game Technology, Information System, Computer Network, Computing, Electronics, and Control, 3(4), 337-344. doi:http://dx.doi.org/10.22219/kinetik.v3i4.708

Receive July 04, 2018; Revise July 14, 2018; Accepted July 18, 2018 
Based on the background has explained before, so done research last project by title "Analysis Tunnelling IPv4 and IPv6 in VolP Network". This research implementation and analysis tunnelling was applied as one of method for connecting between IPv4 and IPv6 with the addiction of VolP services as a real time communication medium.

\section{Research Method}

This research is an experimental research, which analyzes tunneling Internet Protocol Version 6 (IPv6) and Internet Protocol Version 4 (IPv4) in VolP network. Tunneling used is 6to4 tunneling. Implementations on clients use real-time communication media, which is VoIP. Using tunneling method for computer network migration by using two different internet protocols, that is IPv4 and IPv6 in a network infrastructure. The paramaters tested is quality of service that is delay, packet loss, and jitter.

\subsection{Quality of Service (QoS)}

IP QoS Functions are intended to deliver guaranteed as well as differenated internet services by giving network resource and usage control to the network operator. QoS is a set of service requirements to be met by the network in transporting a flow. QoS provide end-to-end service guarantees and policy-based control of an IP network's performance measures, such as resource allocation, switching, routing, packet scheduling, and packet drop mechanism [4].

\subsubsection{Delay}

Delay is the time needed for data to send from source to the destination. Delay can be influenced by distance, physical media, congestion or long processing time. Packet delay, or latency, each hop consists of serialization delay, and switching delay. Category of delay is shown in Table 1.

Table 1. Category of Delay [5]

\begin{tabular}{ll}
\hline Degradation Category & Value of Delay $(\mathrm{ms})$ \\
\hline Very good & $<150$ \\
Good & $150-300$ \\
Medium & $300-450$ \\
Bad & $>450$ \\
\hline
\end{tabular}

\subsubsection{Packet Loss}

Packet loss is a parameter which specifies the number of packets being lost by the network during transmission, which can occur due collision and congestion. Packet drops at network congestion points and corrupted packets on the transmission wire cause packet loss. Packet drops generally occur at congestion points when incoming packet far exceed the queue size limit at the output queue. On the network and this affects all application because re-transmission will reduce overall network efficiency even though the amount of bandwidth is sufficiently available for the application. Packet loss determines the number of packets lost by the network during delivery. Category of packet loss is shown in Table 2.

Table 2. Category of Packet Loss [5]

\begin{tabular}{ll}
\hline Degradation Category & Value of Packet Loss \\
\hline Very good & 0 \\
Good & 3 \\
Medium & 15 \\
Bad & $>25$ \\
\hline
\end{tabular}

\subsubsection{Jitter}

Commonly jitter is called variation of delay, closely related to latency, which shows the value of variation delay in data transmission on the network. Delay queues on routers and switches can affects jitter value. This is caused by variations in queue length, data process time, and the time of reassembling packages at the end of jitter. Category of delay is shown in Table 3.

KINETIK Vol. 3, No. 4, November 2018: 337-344 


\begin{tabular}{ll}
\multicolumn{2}{c}{ Table 3. Category of Jitter [5] } \\
\hline Degradation Category & Value of Packet Loss \\
\hline Very good & 0 \\
Good & $0-75$ \\
Medium & $75-125$ \\
Bad & $>125$ \\
\hline
\end{tabular}

\subsection{Tunneling 6 to 4}

6 to 4 tunneling is a technology in computer networks for the IPv4 Tunneling process to IPv6, where in the use of computer networks (especially the internet) it can easily access IPv6 addresses based on the addresses of IPv4 they use. The workings of this 6 to 4 is by adding an IPv6 packet to the IPv4 package without configuring the Tunnel [6]. To send an IP packet to host 2 , host 1 builds a packet that contains the host IP address 2, then puts it on ethernet. When the multi-protocol router gets the frame, it deletes the IP packet, then inserts it into the payload of the network layer packet and the last address for the WAN address of the multi-protocol router. On arrival in router, the router removes the IP packet and sends it to host 2 in the ethernet frame [7].

\subsection{Internet Protocol (IP)}

Internet Protocol (IP) Address is the address given to all data communication devices that are connected to the internet and it is unique. An IP address is a unique identification address that is owned by each computer and other connected devices in the computer network, as a marker and address of the computer or connected device in question. Based on the amount of capacity of computer network users that can be handled by computers and other devices, the IP address is also divided into two, there are IP version 4 (IPv4) and IP version 6 (IPv6). The IP address currently used is IP version 4 which consists of 32 binary numbers. Since 1994, the use of IPng (next generation) has been recommended which is now referred to as IP version 6 which consists of 128 binary numbers [8].

1. Internet Protocol Version 4 (IPv4)

IPv4 address space is 32 bits wide. IPv4 address space enables 4 billion hosts. IPv4 address space was designed with three classes ( $A, B$ and $C$ ) which makes the address space usage even less efficient. IANA allocates by $1 / 256$ th $(0.4 \%)$ chucks to regional registries, which corresponds to a/8 prefix length or to the leftmost number in an IPv4 address [9].

2. Internet Protocol Version 6 (IPv6)

IPv6 is a new version of the Internet Protocol. It has been designed as an evolutionary, rather than revolutionary. IPv6 space is 128 bits wide each 16 octets each. IPv6 header size is doubled compare the IPv4 header size, but the size of addresses is four times. IPv6 addresses are represented as 8 fields of hexadecimal numbers $(0-F)$, each field representing 16 bits using 4 hexadecimal digits and fields are separated by a colon [9]

\subsection{Voice Over Protocol (VoIP)}

VolP (Voice over Internet Protocol) is one of the voice communication protocols that are popular in the internet world. VolP technology enables voice communication using the internet network. How to use voice communication with VolP is different from Yahoo Messenger. VolP has higher efficiency and effectiveness. The main requirement for VolP communication is a computer that is connected to the internet, and has an audio card connected to the speaker and microphone. With the support of special software (VolP client), between computer users can be connected to each other in a VolP connection with each other [10].

\section{Results and Discussion}

This research implements a network infrastructure by apply the 6to 4 tunnelling transition method in a VolP network. There are 24 clients that will be connected to each other by calling clients to clients (point to point) and conference (point to multipoint). The test was carried out 30 times for each call. This research compares the results of implementing 6 to 4 tunnelling between using IPv4 and IPv6 only.

Based on the Figure 1, there is a network architecture that uses 24 clients that use IPv6, two switches, two MikroTik routers, and a VolP server that uses Asterisk as a server. Each MikroTik Router gets one IPv4 public. The IPv4 obtained was then registered at the Hurricane 
Electric Tunnel broker to get IPv6. Tunnelling is implemented on the MikroTik router. The tunnelling used is 6 to 4 tunnelling.

The client uses the jitsi application to call VoIP each other. IPv4 public is installed on two Mikrotik to register the tunnel broker on Hurricane Electric. Client and server using IPv6 that has been obtained from Hurricane Electric that has been registered from IPv4 public. There is a PC that monitor the network infrastructure that has been implemented. PC monitoring uses Wireshark to see data captured during testing. The data captured on Wireshark is then processed to obtain quality of service values from the network infrastructure that has been implemented.

From the results of testing conducted 30 times with the number of clients as many as 24 . Obtained the results of the QoS value. Testing is done by calling point to point 30 times for 24 clients or 12 client pairs and 30 times for point to multipoint or conference.

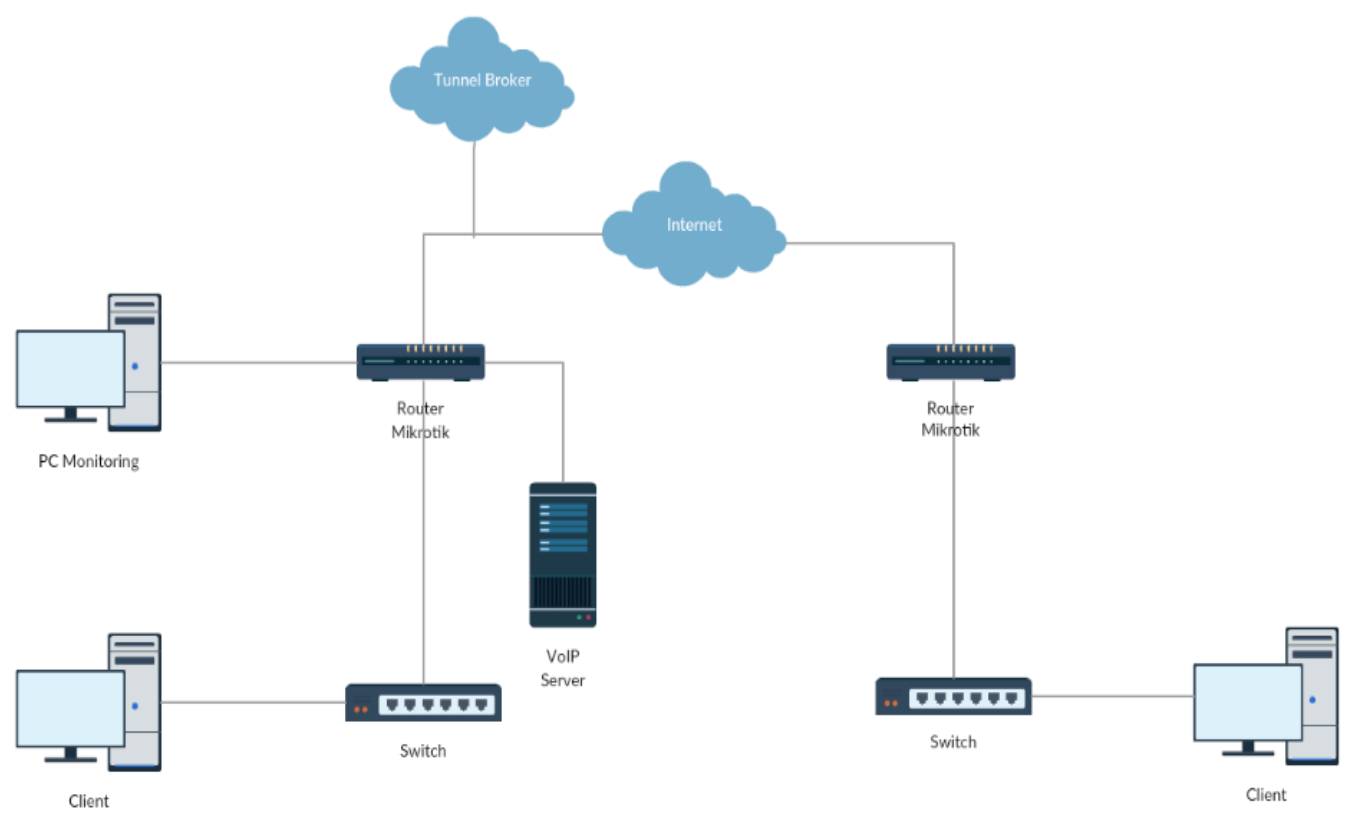

Figure 1. Network Topology

\subsection{Delay}

Delay is the time needed for a package to send from the place of source to the destination. Delay is affected by distance, physical media, etc. Delay is categorized as very good if it gets a value of $<150 \mathrm{~ms}$, if the delay value is $150-300 \mathrm{~ms}$, it is a good and still worthy category.

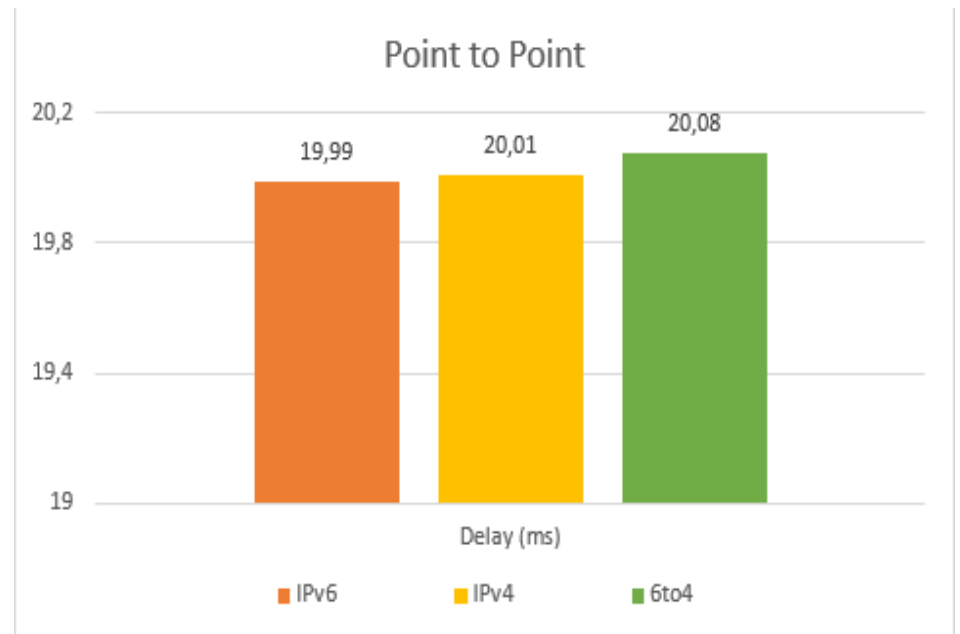

Figure 2. Delay Graphic Point to Point

KINETIK Vol. 3, No. 4, November 2018: 337-344 
In the delay value is obtained the value of the implementation of 6 to 4 which gets the highest delay value by a value of $20.08 \mathrm{~ms}$. The lowest delay value is $19.99 \mathrm{~ms}$ by using an IPv6 clients. Graphic of delay point to point is shown in Figure 2.

\section{Point to Multipoint}

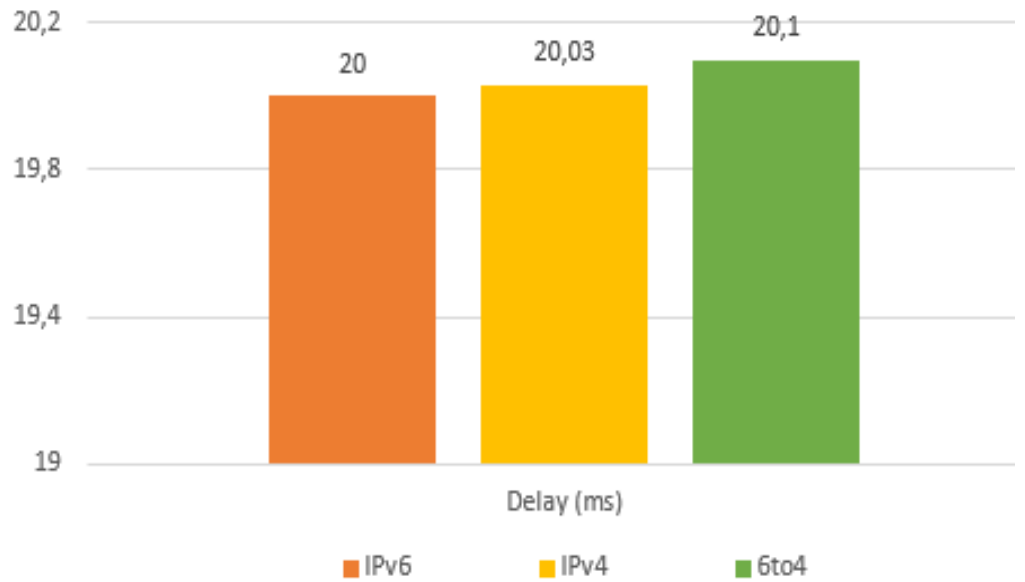

Figure 3. Delay Graphic Point to Multipoint

The value of delay by call point to multipoint results is a higher value than calling point to point. The highest value obtained by implemented 6 to 4 and still the lowest that can use IPv6 with a delay value of 20 ms. Graphic of delay point to multipoint is shown in Figure 3.

\subsection{Packet Loss}

Packet loss is a failure or failure of a packet to get to the destination of the packet from where the packet was sent during the calling process between clients. Packet loss is categorized very well if it has a value of $<1 \%$. However, the value of good packet loss is $2-3 \%$ and is still feasible.

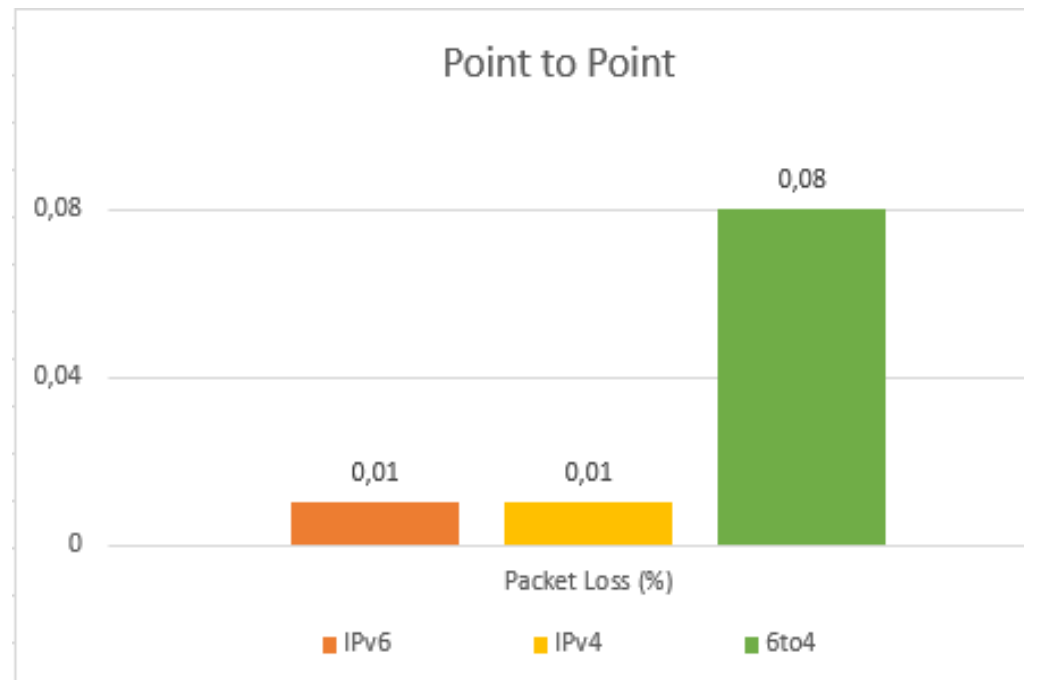

Figure 4. Packet Loss Graphic Point to Point

The lowest value of packet loss is obtained by implementing IPv4 and IPv6. The highest value is obtained by implementing 6 to 4 with a value of $0.08 \%$. Packet loss on IPv4 and IPv6 is very small and there is almost no packet loss. Graphic of packet loss point to point is shown in Figure 4. 
The value of packet loss at the point to multipoint or conference call is the lowest result with the implementation using IPv6. The highest value is obtained by implementing 6to4 tunnelling which a value is $0.1 \%$. The value of IPv6 packet loss is very small as when calling point to point. Graphic of packet loss point to multipoint is shown in Figure 5.

\section{Point to Multipoint}

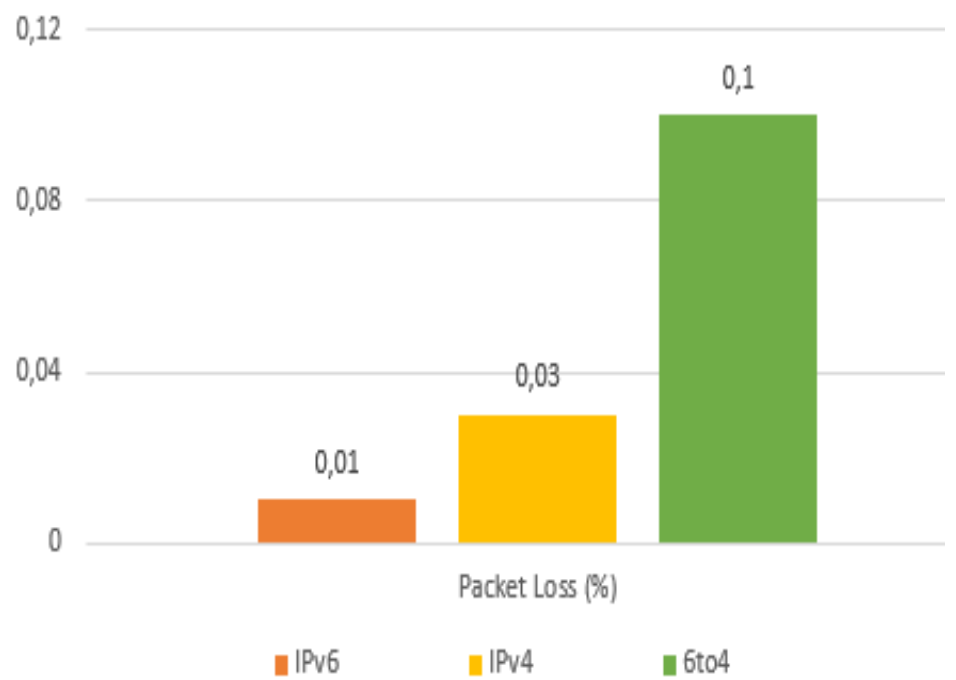

Figure 5. Packet Loss Graphic Point to Multipoint

\subsection{Jitter}

Jitter is a variation of delay that occurs because of the packet arrival time interval at the receiver. The jitter value affects the delay value. To get the jitter value, must first get the delay value first. In addition to delay, high low jitter values are also affected by packages received from each client received [quoting jitter]. A very good jitter value is $5 \mathrm{~ms}$. If the jitter value obtained around $5-75 \mathrm{~ms}$ is categorized as good and still feasible.

\section{Point to Point}

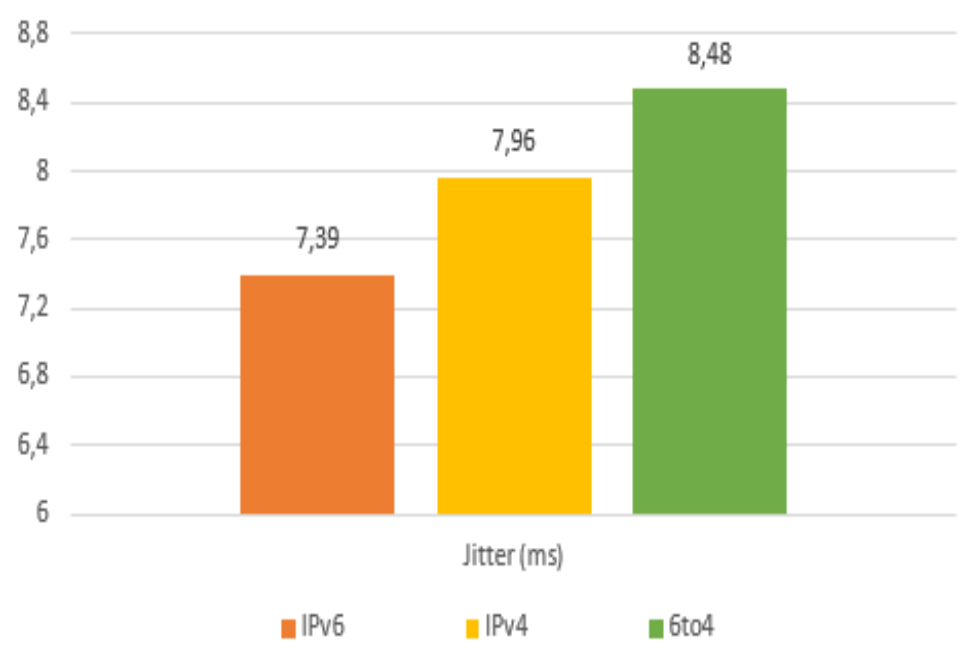

Figure 6. Jitter Graphic Point to Point

Based on testing conducted 30 times with the number of clients is 24 clients. The jitter value obtained by calling point to point generated the lowest value by implementing of IPv6 that is 7,39 $\mathrm{ms}$. The highest jitter value is obtained by implementing 6 to 4 tunneling that is $8,48 \mathrm{~ms}$. Graphic of jitter point to point is shown in Figure 6. 


\section{Point to Multipoint}

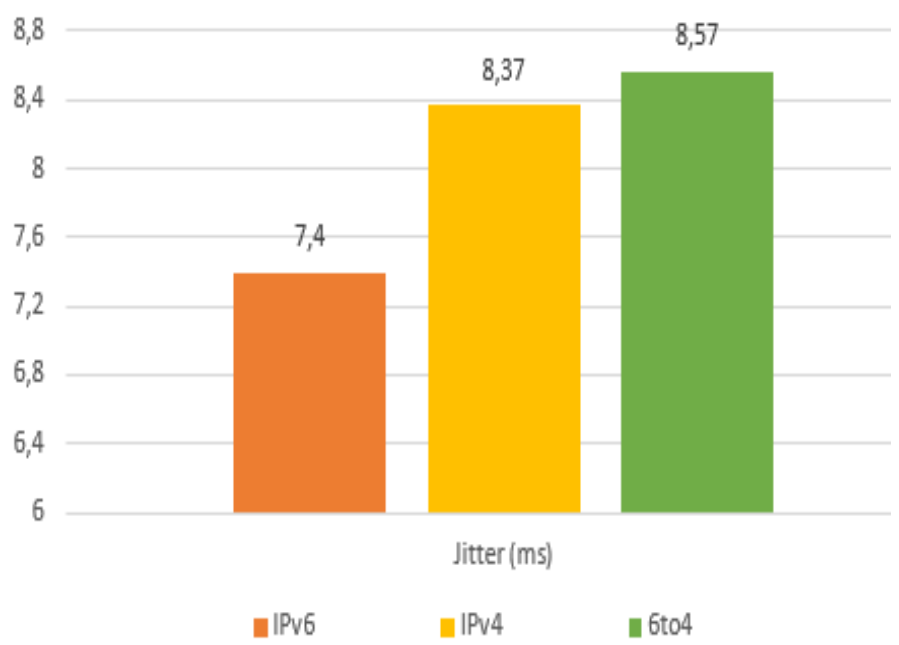

Figure 7. Jitter Graphic Point to Multipoint

The value obtained by calling point to multipoint is higher than by calling point to point. The smallest value is $7,4 \mathrm{~ms}$ by implementin using IPv6. The highest value is 8,57 by implementing 6 to4 tunneling. Graphic of jitter point to point is shown in Figure 7.

\section{Conclusion}

Conclusion from the results of the testing and analysis that has been done, some conclusions can be drawn as follows:

1. Implementation using IPv6 gets the smallest value for delay, jitter, and packet loss.

2. The results of the analysis obtained by the QoS value by calling point to multipoint or conference is higher than by calling point to point.

3. The results of the analysis obtained by implementing using tunneling 6 to 4 obtained high results compared using IPv4 and IPv6 only.

4. The results obtained of using tunneling 6 to 4 are relatively high because IPv6 packets sent are wrapped into the IPv4 form to be able to pass through IPv4 infrastructure which is after arriving at the destination the packet

5. The results by implementing using IPv6 is the lowest because IPv6 is more stable and IPv6 can do routing more simply and quickly.

\section{References}

[1] Siswo Wardoyo, Taufik Ryadi, R. F., "Analisis Performa File Transport Protocol pada Perbandingan Metode Ipv4 Murni, Ipv6 Murni dan Tunneling 6to4 Berbasis Router Mikrotik," Jurnal Nasional Teknik Elektro, 3.2, 1-12, 2014.

[2] Lestari, R. I., "Menganalisa Kinerja antara Metode Tunneling 6 to4 dengan Metode Dual Stack Berbasis Protokol Ipv6 menggunakan Router Mikrotik (Studi Kasus Pt.Time Excelindo)," STMIK Amikom Yogyakarta, 2011.

[3] Artondo, R., "Analisa Dan Implementasi Ipv6 Tunnel Broker untuk Interkoneksi antara Ipv6 dan Ipv4," Universitas Diponegoro, 2011.

[4] Vegesna, S., "Per-Hop Behavior: Congestion Avoidance and Packet Drop Policy," IP Quality of Service,Indianapolis: Cisco Press, 2001.

[5] TIPHON, "Telecommunication and Internet Protocol Harmonization Over Network (TIPHON)," General Aspects of Quality of Service (Qos). Etsi, 2.1.1, 1-37, 1999.

[6] Pratama, I. P., "Handbook Jaringan Komputer," Bandung: INFORMATIKA. 2014.

[7] Tanenbaum, A. S., "Computer Networks," Amsterdam: Prentice-Hall International, Inc, 1996.

[8] Yuhefizar, "10 Jam Menguasai Internet," Jakarta: PT Elex Media Komputindo, 2008.

[9] Blanchet, M. (2006). "Migrating to IPv6," West Sussex: WILEY

[10]Zaki, A., "Berkomunikasi Murah Via Internet," Jakarta: PT Elex Media Komputindo, 2008. 
